\title{
Cortisol and Aldosterone Co-secreting Tumors: A Diagnostic Challenge
}

\author{
Roma Pradhan ${ }^{1}$, Suneel Mattoo ${ }^{2}$, Sushil Gupta ${ }^{3}$, Amit Agarwal $^{4}$
}

\begin{abstract}
The unique subtype of co-secreting adrenal tumor especially aldosterone and cortisol co-secreting tumors presents a special challenge because they present with unexplained clinical constellations, may display unique laboratory test results, and impact postoperative care. Here, we describe three cases of co-secreting adrenal tumor. All our three cases had initial diagnosis of hypercortisolism with atypical features like muscle weakness and hypokalemia which led us to evaluate for hyperaldosteronism. Therefore, we suggest that patients of overt or subclinical Cushing's syndrome who have atypical features should be screened for hyperaldosteronism and similarly patients with primary hyperaldosteronism, especially those with a larger tumor size $(>2.5 \mathrm{~cm})$, should be screened for hypercortisolism to avoid postoperative adrenal crisis.
\end{abstract}

Keywords: Adrenal, Hyperaldosteronism, Hypercortisolism.

World Journal of Endocrine Surgery (2019): 10.5005/jp-journals-10002-1251

\section{INTRODUCTION}

The unique subtype of co-secreting adrenal tumor especially aldosterone and cortisol co-secreting tumors presents a special challenge because they present with unexplained clinical constellations, may display unique laboratory test results, and impact postoperative care. Here we describe three cases of co-secreting adrenal tumor. All our three cases had initial diagnosis of hypercortisolism with atypical features like extreme muscle weakness and persistent hypokalemia which led us to evaluate for hyperaldosteronism.

\section{Cases}

\section{Case 1}

A 38-year-old lady with a history of episode of anxiety, associated with sweating, and palpitation presented to her general practitioner who found her to be having profound hypertension (240/130 $\mathrm{mm} \mathrm{Hg}$ ) and required four hypertensives to control her blood pressures. She was evaluated with a renal artery Doppler and CT angiogram. Computed tomography (CT) showed an adrenal mass $(3 \times 3 \mathrm{~cm})$. Subsequently, the patient was referred to Sanjay Gandhi Post Graduate Institute of Medical Sciences (SGPGI) with a provisional diagnosis of pheochromocytoma. During the evaluation protocol of secondary hypertension, her overnight dexamethasone suppression test (ONDST) and subsequent low dose dexamethasone suppression test (LDDST) were found to be unsuppressible with a normal serum adrenocorticotropic hormone (ACTH) (Table 1). So a diagnosis of subclinical ACTH-independent Cushing's syndrome was considered as she did not have any stigmata of Cushing's syndrome. However, during her evaluation, she was found to be persistently hypokalemic even after stopping diuretics. So she was evaluated for the possibility of having hyperaldosteronism. Serum plasma renin concentration (PRC) was found to be suppressed and serum aldosterone was elevated so that the aldosterone/PRC ratio was calculated to be suggestive of primary hyperaldosteronism (Table 2). Subsequently, the diagnosis of hyperaldosteronism was confirmed by the saline loading test which was positive. \begin{tabular}{l}
\hline 1Department of Endocrine Surgery, Dr Ram Manohar Lohia Institute of \\
Medical Sciences, Lucknow, Uttar Pradesh, India \\
2,4 Department of Endocrine Surgery, Sanjay Gandhi Postgraduate \\
Institute of Medical Sciences, Lucknow, Uttar Pradesh, India \\
${ }^{3}$ Department of Endocrinology, Sanjay Gandhi Postgraduate Institute \\
of Medical Sciences, Lucknow, Uttar Pradesh, India \\
Corresponding Author: Roma Pradhan, Department of Endocrine \\
Surgery, Dr Ram Manohar Lohia Institute of Medical Sciences, \\
Lucknow, Uttar Pradesh, India, Phone: +919732221166 , e-mail: \\
drromapradhan@yahoo.com \\
How to cite this article: Pradhan R, Mattoo S, Gupta S, Agarwal A. Cortisol \\
and Aldosterone Co-secreting Tumors: A Diagnostic Challenge. World J \\
Endoc Surg 2019;11(1):28-30. \\
Source of support: Nil \\
Conflict of interest: None
\end{tabular}

Contrast enhanced computed tomography (CECT) (Fig. 1) was done with washout studies which was suggestive of adenoma. So with the preoperative diagnosis of a co-secreting adrenal mass, she was taken up for laparoscopic right adrenalectomy under steroid cover. A soft encapsulated, golden yellow tumor of size $4 \mathrm{~cm}$ was dissected along with attached adrenal gland. Postoperative recovery was uneventful. Microscopic features were suggestive of two different types of cells-clear cells and compact cells representing two different layers of the adrenal gland (Fig. 2).

\section{Case 2}

A 40-year-old lady from Nepal presented with profound muscle weakness and weight gain since the last 3 years. The patient was a known hypertensive since the last 3 years which was poorly controlled on two antihypertensives. Ultrasonography (USG) abdomen and CECT abdomen showed $2.5 \times 2.1 \mathrm{~cm}$ heterogeneous right adrenal lesion. A hormonal evaluation revealed ACTHindependent hypercortisolism so a diagnosis of cortisolproducing adenoma was made. However, she had unexplained profound proximal muscle weakness and progressive weakness of lower limbs. Neurological evaluation ruled out a neurological 
Table 1: Biochemical evaluation

\begin{tabular}{llll}
\hline Test & Patient 1 & Patient 2 & Patient 3 \\
\hline ONDST & $78 \mathrm{nmol} / \mathrm{L}$ (unsuppressed) & $208 \mathrm{nmol} / \mathrm{L}$ (unsuppressed) & $161 \mathrm{nmol} / \mathrm{L}$ (unsuppressed) \\
LDDST & $135 \mathrm{nmol} / \mathrm{L}$ (unsuppressed) & $174 \mathrm{nmol} / \mathrm{L}$ (unsuppressed) & $268 \mathrm{nmol} / \mathrm{L}$ (unsuppressed) \\
8 am ACTH & $1.02 \mathrm{pmol} / \mathrm{L}(\downarrow)$ & $1.10 \mathrm{pmol} / \mathrm{L}(\downarrow)$ & $1.74 \mathrm{pmol} / \mathrm{L}(\downarrow)$ \\
$24-$ hour UMN & $64 \mu \mathrm{g}$ & $44 \mu \mathrm{g}$ & $64 \mu \mathrm{g}$ \\
24-hour UNMN & $425 \mu \mathrm{g}$ & $209 \mu \mathrm{g}$ & $343 \mu \mathrm{g}$ \\
\hline
\end{tabular}

Table 2: Biochemical tests for hyperaldosteronism

\begin{tabular}{llll}
\hline Test & Patient 1 & Patient 2 & Patient 3 \\
\hline $\mathrm{K}^{+}$ & 3.0 & $4.2 \mathrm{mEq} / \mathrm{dL}$ & 3.1 \\
Aldosterone (PAC) & Elevated $(\uparrow)$ & Elevated $(\uparrow)$ & Elevated $(\uparrow)$ \\
PRA & Suppressed & Suppressed & Suppressed \\
Aldo/PRA ratio (ARR) & Elevated $(\uparrow)$ & Elevated $(\uparrow)$ & Elevated $(\uparrow)$ \\
Saline loading test & Positive & Positive & Positive \\
\hline
\end{tabular}



Fig. 1: $3.5 \times 2.4 \times 3.2 \mathrm{~cm}$ heterogeneous hypodense lesion in the right suprarenal space arising from the lateral limb of right adrenal opposite adrenal normal attenuation value (HU) plain: 65, portal venous 140 , delayed 90 absolute washout $=60 \%$ relative washout $=35.7 \%$

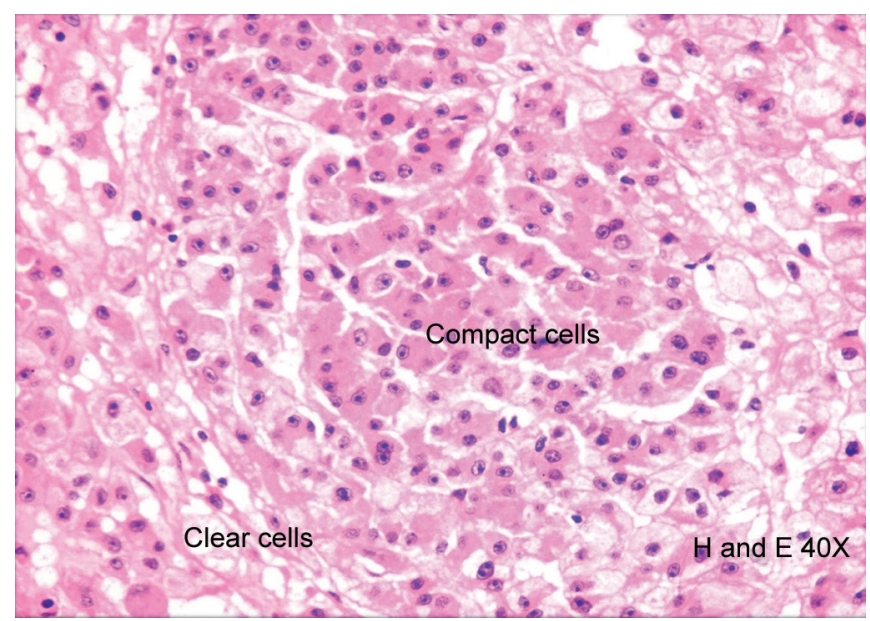

Fig. 2: Histopathology (40x) aldosterone secreting clear cells are larger and with clear cytoplasm cortisol-secreting compact cells are smaller and eosinophilic

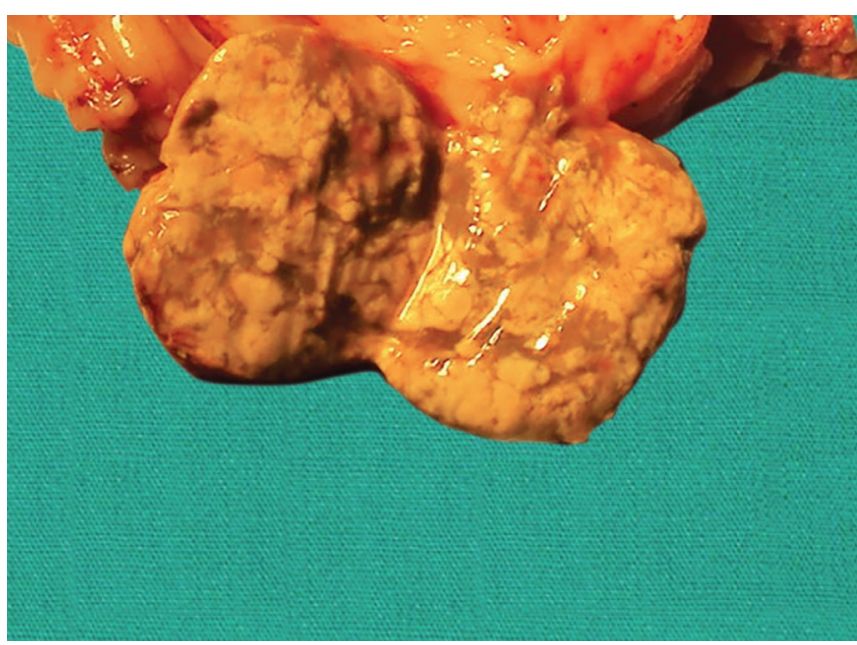

Fig. 3: Specimen cut section: golden yellow admixed with islet-like distribution of $\tan /$ brown portion

etiology of weakness so the possibility of hyperaldosteronism was also thought of and she was then evaluated by serum aldosterone and plasma renin activity (PRA). The serum aldosterone/PRC ratio was found to be elevated and subsequent saline loading test was positive. The diagnosis was then revised to a co-secreting adrenal tumor. The patient underwent laparoscopic adrenalectomy under steroid cover. The specimen weighed $30 \mathrm{~g}$ with a $3.0 \times 2.5 \times 2.5 \mathrm{~cm}$ tumor. The cut section revealed golden yellow tumor admixed with tan brown portion (Fig. 3) histopathology showing nodules composed of two types of cells, i.e., clear and compact cells consistent with aldosterone and cortisol co-secreting adrenal adenoma.

\section{Case 3}

A 35-year-old lady presented with complaints of anxiety and palpitation for 10 months with a history of migraine. On evaluation, she was found to have high blood pressure 170/100 $\mathrm{mm} \mathrm{Hg}$ and was subsequently started on the calcium channel blocker. During the evaluation, she underwent an USG of the abdomen which showed $7 \times 5 \times 4.5 \mathrm{~cm}$ left adrenal lesion and, hence, referred to SGPGI for further evaluation and management. She had two uneventful lower segment cesarian section (LSCS). A clinical diagnosis of secondary hypertension with the possibility of pheochromocytoma was made. However, on biochemical evaluation (Table 1), her 24-hour urinary metanephrine (MN) and normetanephrines (NMN) were found to be normal but her ONDST and subsequently LDDST both were unsuppressible with normal ACTH. Hence, her diagnosis was revised to subclinical Cushing's syndrome with the possibility of adrenocortical carcinoma in view of large size. During her first admission, her serum potassium was found to be continuously low $(3.1,3.0,3.6,3.4$, and 3.0) and she had to be put on oral potassium liquid. She was asked leading questions about the use of diuretic 
or periodic paralysis which was negative. Subsequently, her plasma aldosterone was found to be elevated with suppressed PRC leading to a high aldo/PRA ratio. The primary hyperaldosteronism was confirmed by a positive saline loading test. Imaging studies included a CECT which showed a heterogeneously enhancing mass (the largest dimension of $6.8 \mathrm{~cm}$ ) involving the left adrenal gland abutting the tail of the pancreas and spleen with a few areas of arterial enhancement with a progressive enhancement on the venous phase, with slow wash out $(<45 \%)$. There were no foci of calcification/macroscopic fat or significant intra-abdominal lymphadenopathy. The fluorodeoxyglucose-positron emition tomography (FDG PET) scan was done in view of the possibility of adrenocortical carcinoma (ACC) and it revealed a metabolically active left suprarenal mass lesion. So the preoperative diagnosis was of a left adrenal mass with primary hyperaldosteronism and hypercortisolism with suspicion of malignancy because of multihormone secretion and size of the lesion. Open transperitoneal adrenalectomy was performed under a perioperative cover of steroid. A well-encapsulated mass, heterogeneous with fleshy hemorrhagic areas measuring $8 \mathrm{~cm}$, was removed. The specimen weighed $88 \mathrm{~g}$. Histopathology was consistent with adrenocortical adenoma with the modified Weiss score of less than 2 (necrosis: absent, capsular/venous invasion: absent, mitoses: absent, clear cell area $>25 \%$ ). The patient was continued with postoperative supplementation of mineralocorticoid and prednisolone. The postoperative period was uneventful. She was discharged on tab prednisolone $3.75 \mathrm{mg}(2.5+1.25)$ and calcium channel blocker. She is still on the same dose of steroid after inadequate response on the Synacthen test at 6 months.

\section{Discussion}

The hormonal evaluation of an incidentaloma is still controversial even though there are many practice guidelines on various diagnostic tests for confirming the diagnosis in a suspected case of Cushing's syndrome or hyperaldosteronism. The unique subtype of co-secreting adrenal tumor especially aldosterone and cortisol co-secreting tumors present a special challenge because they present with unexplained clinical constellations, may display unique laboratory test results, and impact postoperative care.

Subclinical Cushing's syndrome which is a state of cortisol hypersecretion without overt signs or symptoms of Cushing's syndrome is not so rare as previously thought. ${ }^{1}$ Hence, all the patients with adrenal mass should be evaluated to rule out its functionality. In fact, all patients with adrenal mass should be evaluated biochemically for evidence of hypercortisolism, aldosteronism (if hypertensive), and the presence of pheochromocytoma.

The first report of a patient with primary aldosteronism and Cushing's syndrome due to an aldosterone-producing and cortisolproducing adenoma was obtained in 1977 by Hogan et al. ${ }^{2}$ In the past decade, several case reports, series, and review have been published in the literature. ${ }^{3-5}$

Subclinical hypercortisolism in unilateral primary aldosteronism may generally be underdiagnosed due to incomplete investigations performed as many of these patients will not undergo the overnight dexamethasone suppression test. However, Piaditis et al. have reported a high percentage of $12.1 \%$ of co-secreting tumors in 83 unilateral adrenal incidentalomas. These patients are at risk of developing more cardiovascular and metabolic complications because of excess of two steroids in the body. ${ }^{6}$ Further, patient may land up in adrenal crisis postoperatively if the diagnosis is not made preoperatively. So for any adrenal mass evaluation, it is important that we do screening tests (ONDST and 24-hour urinary MN and NMN). Hence, we recommend the dexamethasone suppression test in all patients of confirmed PA to rule out hypercortisolism. This is especially important in patients of primary aldosteronism with a large adrenal mass because A/ACAs are usually larger than adenomas with only primary aldosteronism (PA). All our three patients had a size more than $2.5 \mathrm{~cm}$. Späth et al. ${ }^{3}$ recently reported that we should consider an aldosterone producing adenoma (APA) with co-secretion of cortisol if a patient has (1) PA and an adenoma that is larger than $2.5 \mathrm{~cm}$, (2) cortisol that is nonsuppressible with overnight low-dose dexamethasone, or (3) grossly elevated serum levels of hybrid steroids, such as $18-\mathrm{OH}-\mathrm{F}$. Another important laboratory finding in patients with APA/CS is low levels of ACTH. ${ }^{1}$

In the review by Späth et al., ${ }^{3}$ more than two-third of reported patients in the literature were females. All our three patients in the series were female 40 or less than 40 years, though this female preponderance is not consistently seen in cases of primary aldosteronism. Only one of our three patients had clinical Cushing's syndrome, the rest were cases of subclinical Cushing's syndrome. All of our cases were diagnosed preoperatively as we have a set protocol for evaluation of adrenal mass. In the review by Späth et al., ${ }^{3}$ two patients were retrospectively diagnosed with Cushing's syndrome due to the adrenal crisis in which the patient landed postoperatively.

All our patients had hypokalemia with raised aldosterone renin ratio (ARR) (aldo PRA ratio) and positive saline loading test. In the literature review, the saline loading test was positive in $60 \%$ of cases. The other cases in the literature were confirmed with the fludrocortisone suppression test. ${ }^{3}$

\section{ConClusion}

Based on our experience, we would suggest that patients of overt or subclinical Cushing's syndrome who have atypical features should be screened for hyperaldosteronism and similarly patients with primary hyperaldosteronism especially those with a larger tumor size $(>2.5 \mathrm{~cm})$ should be screened for hypercortisolism to avoid postoperative adrenal crisis.

\section{References}

1. Hiraishi K, Yoshimoto T, et al. Clinicopathological features of primary aldosteronism associated with subclinical Cushing's syndrome. Endocr J 2011;58(7):543-551.

2. Hogan MJ, Schambelan M, et al. Concurrent hypercortisolism and hypermineralocorticoidism. Am J Med 1977;62:777-782.

3. Späth $\mathrm{M}$, Korovkin S, et al. Aldosterone- and cortisol-co-secreting adrenal tumors: the lost subtype of primary aldosteronism. Eur J Endocrinol 2011;164:447-455. DOI: 10.1530/EJE-10-1070.

4. Vicennati V, Repaci A, et al. Combined aldosterone and cortisol secretion by adrenal incidentaloma. Int J Surg Pathol 2012;20:316-319. DOI: 10.1177/1066896911427036.

5. Yamada M, Nakajima Y, et al. KCNJ5 mutations in aldosterone- and cortisol-co-secreting adrenal adenomas. Endocr J. 2012;59(8):735-741.

6. Nakajima Y, Yamada M, et al. Cardiovascular complications of patients with aldosteronism associated with autonomous cortisol secretion. JClin Endocrinol Metab 2011;96:2512-2518. DOI: 10.1210/jc.2010-2743. 